\title{
Aurora Kinase A expression is associated with lung cancer histological-subtypes and with tumor de-differentiation
}

\author{
Marco Lo lacono ${ }^{*}$, Valentina Monica, Silvia Saviozzi, Paolo Ceppi, Enrico Bracco, Mauro Papotti and \\ Giorgio V Scagliotti
}

\begin{abstract}
Background: Aurora kinase $\mathrm{A}(A \cup R K A)$ is a member of serine/threonine kinase family. Several kinases belonging to this family are activated in the G2/M phase of the cell cycle being involved in mitotic chromosomal segregation. AURKA overexpression is significantly associated with neoplastic transformation in several tumors and deregulated Aurora Kinases expression leads to chromosome instability, thus contributing to cancer progression. The purpose of the present study was to investigate the expression of AURKA in non small cell lung cancer (NSCLC) specimens and to correlate its mRNA or protein expression with patients' clinico-pathological features.
\end{abstract}

Materials and methods: Quantitative real-time PCR and immunohistochemistry analysis on matched cancer and corresponding normal tissues from surgically resected non-small cell lung cancers (NSCLC) have been performed aiming to explore the expression levels of AURKA gene.

Results: AURKA expression was significantly up-modulated in tumor samples compared to matched lung tissue $(p<0.01$, mean $\log 2(F C)=1.5)$. Moreover, $A U R K A$ was principally up-modulated in moderately and poorly differentiated lung cancers $(p<0.01)$, as well as in squamous and adenocarcinomas compared to the non-invasive bronchioloalveolar histotype $(p=0.029)$. No correlation with survival was observed.

Conclusion: These results indicate that in NSCLC AURKA over-expression is restricted to specific subtypes and poorly differentiated tumors.

\section{Background}

Aurora kinase A (AURKA) is a member of serine/threonine kinase family: homologous to both the Drosophila aurora and Saccharomyces cerevisiae Ipl1 kinase families. It plays an important role in completing mitotic events such as centrosome separation, bipolar spindle assembly, chromosome segregation and cytokinesis [1]. Aurora A expression is cell-cycle regulated. Indeed its mRNA, protein levels and kinase activity are low in the G1/S phase; it accumulates during G2/M and decreases rapidly after mitosis. Aurora A protein is localized in the centrosomes of interphase cells and in the spindle of mitotic cells. Ectopic expression of Aurora $A$ leads to an increase in centrosome numbers, causes catastrophic loss or gain of chromosomes, and results in either cell

\footnotetext{
* Correspondence: marco.loiacono@unito.it

Department of Clinical and Biological Sciences, University of Turin, Turin, Italy
}

death or survival through malignant transformation [2]. Over-expression of AURKA has been detected in many tumor cells and tissues, such as breast, gastric, colorectal, bladder, pancreatic, ovarian, prostate and lung cancers [3-8]. Previous data has pointed out that AURKA over-expression is associated with the carcinogenesis and/or drug resistance in many human malignant tumors. Indeed, AURKA phosphorylates p53, abrogates both p53 DNA binding and transactivation activities. In such context, AURKA overrides the apoptosis and cell cycle arrest induced by cisplatin and $\gamma$-irradiation, respectively [9].

AURKA over-expression was also correlated with clinical stage and metastasis and its inhibitions to reduce cell invasion in vivo $[10,11]$. However, AURKA expression was involved in the epithelial-mesenchimal transition (EMT) of nasopharyngeal carcinoma. Indeed, the inhibition of 
AURKA suppresses invasion and increases the expression of different epithelial markers [12].

The aim of the present study was to investigate the AURKA expression levels in lung tumors and their corresponding morphologically normal lung tissues, obtained from the same resected lobe in patients with early stage NSCLC. Correlation between AURKA expression, patients' clinicopathological features and survival was assessed.

\section{Materials and methods}

\section{Patients and samples}

Frozen primary lung tumor and corresponding non-neoplastic lung specimens of 83 consecutive NSCLC patients who underwent radical surgery at the San Luigi Hospital, Division of Thoracic Surgery, between December 2003 and March 2005, were analyzed.

Patients (64 males and 19 females) had a median age of 67 years (range 40 to 82 years) and no patient received either pre-operative or post-operative chemo and/or radio-therapy according to the institutional treatment policy for resectable rescue in those years. Histological examination was performed on formalin-fixed tissues in all cases and tumors were diagnosed and classified according to the WHO classification [13] as follows:

40 adenocarcinomas (ADC); 30 squamous cell carcinomas (SQC); 4 large cell carcinomas (LCC); and 9 bronchiolo-alveolar carcinoma/adenocarcinoma in situ (BAC/AIS). Differentiation grade (grade 1: 16, grade 2: 29, grade 3: 38), p T status (pT1: 7, pT2: 59, pT3: 9, pT4: 8) and $\mathrm{pN}$ status (pN0: 52, pN1: 13, pN2: 18) were also recorded. According to the TNM classification for solid tumors [14], 41 cases had a pathological stage I; 15 stage II; 24 stage III; and 3 stage IV. Follow up data was available for all cases. Informed consent was obtained from each patient and the study was approved by the Institutional Review Board of the San Luigi Hospital. All samples were de-identified and cases anonymized by a pathology staff member not involved in the study. Clinical parameters were compared and analyzed through coded data.

\section{RNA extraction, cDNA synthesis and Qpcr}

RNA was extracted from 15-25 mg and 60-80 mg of tumor and normal lung tissue specimens, respectively. Genomic DNA contamination was removed by DNAseI treatment (Promega). TotRNA was then quantified with an Agilent 2100 Bioanalyzer (Agilent Technologies, Palo Alto, CA) and stored at $-80^{\circ} \mathrm{C}$. Two $\mu \mathrm{g}$ totRNA were retro-transcribed with random hexamer primers and Multiscribe Reverse transcriptase (High Capacity cDNA Archive Kit, Applied Biosystems, Foster City, CA), in accordance with manufacturer's suggestions.
Expression levels of AURKA and of reference genes $P O L R 2 B$ and ESD were evaluated with SYBR technology with optimized PCR conditions and primer concentrations. Primer sequences were as follows: AURKA.FW: GAGATTTTGGGTGGTCAGTAGATG, AURKA.RW: TAGTCCAGCGTGCCACAGAGA, ESD.FW:TGTTGTC ATTGCTCCAGATACCA, ESD.RW:CCCAGCTCTCAT CTTCACCTTT, POLR2B.FW:CCTGATCATAACCAG TCCCCTAGA,OLR2B.RW:GTAAACTCCCATAGCCT GCTTACC.

Melting curve analysis and efficiency evaluations were performed for all the amplicons. Quantitative PCR (qPCR) was carried-out on an ABI PRISM 7900 HT Sequence Detection System (Applied Biosystems) in 384-well plates assembled by Biorobot 8000 (Qiagen, Germantown, ML). Reactions were performed in a final volume of $20 \mu \mathrm{l}$. All qPCR mixtures contained $1 \mu \mathrm{l}$ of cDNA template, $1 \mathrm{X}$ SYBR Universal PCR Master Mix (2x) (Applied Biosystems). Cycle conditions were as follows: after an initial 2-min hold at $50^{\circ} \mathrm{C}$ to allow AmpErase-UNG activity, and 10 minutes at $95^{\circ} \mathrm{C}$, the samples were cycled 40 times at $95^{\circ} \mathrm{C}$ for 15 seconds and $60^{\circ} \mathrm{C}$ for 1 minute. Baseline and threshold for $\mathrm{Ct}$ calculation were set-up manually with the ABI Prism SDS 2.1 software.

\section{Immunohistochemistry}

Formalin-fixed paraffin-embedded tissues were cut into 4 $\mu \mathrm{m}$ thick sections and collected onto charged slides for immunohistochemical staining. After de-paraffination and rehydration through graded alcohols and phosphate-buffered saline ( $\mathrm{pH}$ 7.5), the endogenous peroxidase activity was blocked by incubation with absolute methanol and $0.3 \%$ hydrogen peroxide for 15 minutes. Sections were incubated at the optimal conditions with the following primary antibodies:

(1) mouse monoclonal antibody anti-Ki67 (1:300; MIB-1, DakoCytomation, Glostrup, Denmark); (2) Mouse monoclonal AURKA (1:200; H00006790-M01, Abnova, Taipei, Taiwan).

Immunoreaction was revealed by a dextran-chain (biotin-free) detection system (EnVision; DakoCytomation), using 3,3'-diaminobenzidine (DAB; DakoCytomation) as a chromogen. The sections were lightly counterstained with haematoxylin. Negative control reactions were obtained by omitting the primary antibody. Ki67 proliferation index was calculated as the percentage of positive nuclei amongst at least 200 nuclei counted at high magnification in areas of highest labeling.

\section{Statistical analysis}

AURKA mRNA Ct values, calculated by Applied Biosystems SDS2.1 software, were normalized by subtraction of the geometric mean obtained between $\mathrm{Ct}$ for two internal controls, $P O L R 2 B$ and $E S D$, generating $\triangle \mathrm{Ct}$ 
values. Differential $A U R K A$ transcript expression between $\triangle \mathrm{Ct}$ values for tumor and corresponding normal tissue samples were evaluated using t-test for paired data and expressed by the formula: $\Delta \Delta \mathrm{Ct}=-(\Delta \mathrm{Ct}$ cancer - $\Delta$ Ct normal) corresponding to $\log _{2}$ [fold change]. Protein and mRNA expression levels have been dichotomized into two groups of "high" and "low" expression using median value as threshold cut-off. For AURKA staining intensity, percentage of cells with nuclear expression and $\mathrm{H}$ score (intensity $\mathrm{x} \%$ cells positive) were evaluated. The association between $\Delta \Delta \mathrm{Ct}$ and clinico-pathological variables was evaluated using the Kruskal-Wallis test. Overall survival time was calculated from the date of surgery to death or last follow-up date. Cox regression was used in the univariate survival analysis to determine the association of AURKA modulation with overall survival. Statistical analysis was performed using R statistical software [15].

\section{Results}

In our NSCLC patients' cohort, we observe a higher $A U R K A$ transcript level in tumor specimens against the corresponding morphologically normal adjacent lung tissues (Figure.1. $\mathrm{p}<0.01$, mean $\log _{2}(\mathrm{FC})=1.5$ ). AURKA mRNA level showed variability according to histological subtypes with the highest expression in squamous cell carcinomas (mean $\log _{2}(\mathrm{FC})=2.7, \mathrm{p}<<0.01$ ) and in large cell carcinomas (mean $\log _{2}(\mathrm{FC})=2.25$, $\mathrm{p}<<0.01$ ) followed by adenocarcinomas (mean $\log _{2}(\mathrm{FC})=1.5, \mathrm{p}=$ 0.02 ) and bronchioloalveolar/in situ carcinomas (mean $\log _{2}(\mathrm{FC})=0.28, \mathrm{p}=0.4$ ) (Figure.2, panel A). The lowest

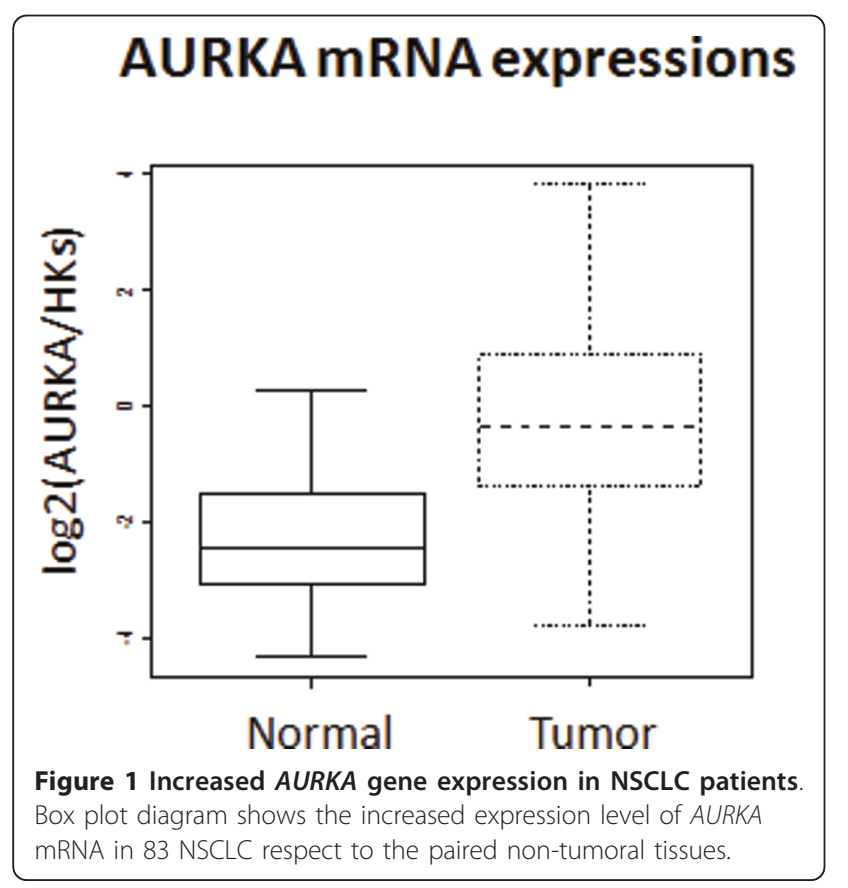

expression observed in BAC histotypes was significantly different compared to the other tumor subtypes ( $\mathrm{p}=$ 0.029). Moreover, AURKA mRNA was significantly over-expressed in poor (grade III) or moderately differentiated (grade II) lung cancer specimens compared to well-differentiated cases (grade I) (Figure.2, panel B, p < 0.01). No correlation between AURKA gene expression and patient's age $(\mathrm{p}=0.59)$, sex $(\mathrm{p}=0.12)$, TNM stage ( $\mathrm{p}=0.39)$, or smoking status $(\mathrm{p}=0.62)$ and, with overall survival rates $(\mathrm{p}=0.39)$ was identified.

The AURKA protein expression was investigated by immunohistochemistry (IHC) in 30 NSCLC patients specimens showing nuclear compartment immunoreactivity in $97 \%$ samples. Both the associations previously identified between AURKA transcript expression and tumors histological subtypes and differentiation grade were also confirmed at protein level (Table 1 and 2, Figure 3).

Moreover, since AURKA expression is increased during the $\mathrm{G} 2 / \mathrm{M}$ phase cell cycle, we also evaluated the correlation between AURKA expression and proliferation marker ki67. AURKA mRNA expression and ki67 were correlated only in $33 \%$ of tumors samples ( $\mathrm{p}=$ 0.055), and this association was slightly increased for AURKA protein expression (H score: $40 \%, \mathrm{p}=0.029, \%$ positive cells: $38 \%, \mathrm{p}=0.04)$.

$A U R K A$ expression is involved in the epithelialmesenchimal transition (EMT) and invasion of nasopharyngeal carcinoma [12]. To test the hypothesis of a similar mechanism in lung cancer we evaluated the effect of AURKA inhibition in NSCLC cell lines (H522, H1299 and Calu1). The FACS analysis reveals that the inhibition of AURKA activity, by the specific inhibitor PHA739358, slightly increases the expression of E-cadherin (Additional file 1 Figure S1. Panel A), although this effect was transcriptionally independent. Indeed, the E-Cadherin gene expression (Additional file 1 Figure S1. Panel B) was unaffected in the H522 cell line by AURKA transcript silencing or by AURKA enzymatic inhibition, using the specific inhibitor PHA-739358. Furthermore, the inhibition of AURKA activity does not modify the normal cellular migration of H522 and Calu1 cell lines, while significantly stimulates the motility of high invasive H1299 cell line ("p $<0.05$, Additional file 1 Figure S1. Panel C). The experimental procedures utilized in these experiments were illustrate in Additional file 2.

\section{Discussion}

In the present study we evaluated AURKA expression in NSCLC showing that at both transcript and protein levels, the AURKA expression was significantly upmodulated in NSCLC tumor samples compared to matched lung normal tissue $(\mathrm{p}<0.01$, mean $\log 2(\mathrm{FC})=$ 1.5). The low correlation observed between AURKA 


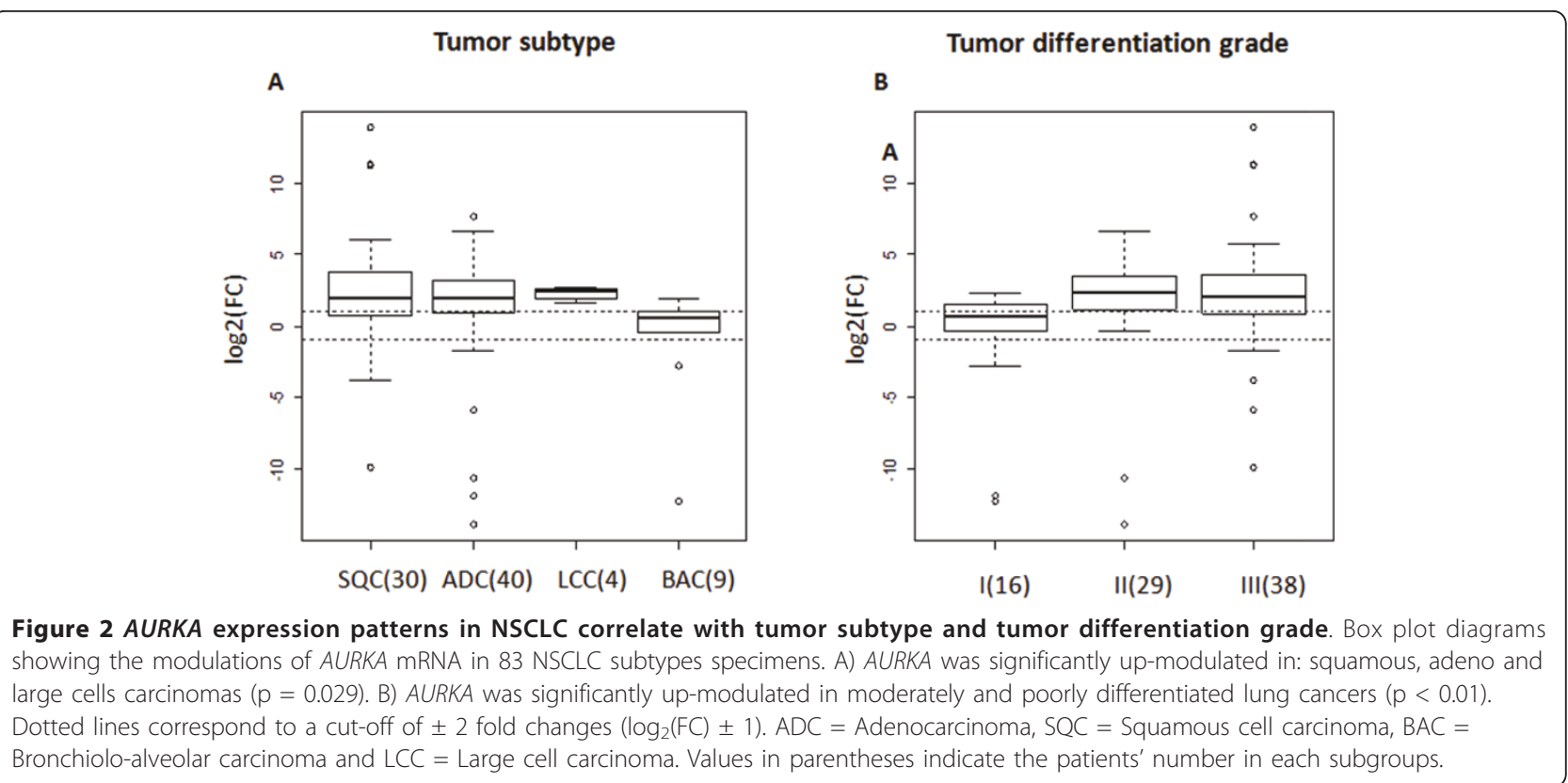

expression and ki67 proliferation marker (33-40\% with transcript and protein respectively) assert that the AURKA up-modulation identified in NSCLC was not only due to a higher proliferation rate but suggests its involvement in cancer pathogenesis. Indeed, we observed a significantly higher AURKA transcript expression in poorly and moderate differentiated tumors compared to well differentiated ones (Figure.2, panel B, $\mathrm{p}<0.01$ ). Our data is in agreement with a previous report by $\mathrm{Xu}$ et al. [8] who identified the AURKA protein over-expression in poorly differentiated lung cancer. Together, this data supports the hypothesis that chromosomal instability associated with progression of lung tumors could be related with AURKA deregulation. Xu et al. observed the AURKA protein over-expression in grade III tumors. We also identified the AURKA mRNA over-expression in moderately differentiated tumors. This result may indicate a better sensibility of qPCR analysis respect to IHC for identifying AURKA deregulation and the evaluation of AURKA mRNA could be a useful biomarker to identify tumor de-differentiation at early levels.

Table 1 Correlation between AURKA protein expression levels and transcript analysis read-out.

\begin{tabular}{ccccccc}
\hline AURKA & \multicolumn{3}{c}{$\begin{array}{c}\text { cells with protein } \\
\text { expression }\end{array}$} & $\begin{array}{c}\text { H score protein } \\
\text { exp }\end{array}$ \\
\hline mRNA exp tumor & High & Low & High & Low & \\
High & 10 & 4 & $\mathrm{p}=0.03$ & 10 & 4 & $\mathrm{p}=0.03$ \\
Low & 5 & 11 & & 5 & 11 & \\
\hline
\end{tabular}

Our data clearly showed the different histological subtypes of NSCLC exhibited in different levels of AURKA modulation ordered from the highest to the lowest as follows: SQC (mean $\left.\log _{2}(\mathrm{FC})=2.7, \mathrm{p}<<0.01\right)$, LCC (mean $\left.\log _{2}(\mathrm{FC})=2.25, \mathrm{p}<<0.01\right), \mathrm{ADC}\left(\right.$ mean $\log _{2}(\mathrm{FC})=1.5 \mathrm{p}=$ 0.02 ) and $\mathrm{BAC}\left(\right.$ mean $\log _{2}(\mathrm{FC})=0.28, \mathrm{p}=0.4$ ) (Figure.2, panel A). Interestingly, the same histological subtypes ranking was reported also for p53 mutations status [16]. It has been demonstrated that the effect of Aurora-A overexpression on tetraploidisation and centrosome amplification depends on the p53 status [17]. Moreover, Tonon et al. suggest a higher grade of genomic instability in SCQ than in ADC [18]. This data, further underlines the tight connection between AURKA over-expression, p53 functions and the genomic instability in NSCLC.

AURKA expression is involved in the epithelialmesenchimal transition (EMT) of nasopharyngeal carcinoma [12] and its inhibition reduces cell invasion in hepatocellular and in head/neck squamous cell carcinoma $[10,11]$. To test the hypothesis of a similar mechanism in lung cancer we evaluated the effect of AURKA inhibition in non small carcinoma cell lines (H522, H1299 and Calu1).

E-cadherin based junctional complexes keep epithelial cells in a stationary, non-motile state and disruption of this cell-cell adhesion mechanism is a crucial step for tumour invasion. Down-regulation of E-cadherin is one of the main changes occurring in pathological EMTs and causes destabilization of the epithelial architecture [19]. Indeed, E-cadherin acts as a tumour suppressor against invasion and metastasis, and its function is impaired during the malignant progression of most carcinomas 
Table 2 Correlation between AURKA protein expression levels and transcript analysis stratifying by subtypes and NSCLC differentiation grade

\begin{tabular}{|c|c|c|c|c|c|c|}
\hline \multirow[b]{2}{*}{ Tumor subtype } & \multicolumn{2}{|c|}{ mRNA expression } & \multicolumn{2}{|c|}{$\%$ cells protein exp } & \multicolumn{2}{|c|}{ H score protein exp } \\
\hline & High & Low & High & Low & High & Low \\
\hline ADC & 6 & 6 & 6 & 6 & 6 & 6 \\
\hline BAC/AIS & 1 & 5 & 1 & 5 & 2 & 4 \\
\hline SQC & 7 & 5 & 8 & 4 & 7 & 5 \\
\hline Differentiation grade & High & Low & High & Low & High & Low \\
\hline I & 2 & 7 & 3 & 6 & 4 & 5 \\
\hline$\|$ & 5 & 5 & 3 & 7 & 3 & 7 \\
\hline III & 7 & 4 & 9 & 2 & 8 & 3 \\
\hline
\end{tabular}

including lung cancer [20]. We report that $A U R K A$ expression/activity in lung cancer cell lines does not regulate the transcriptional level of E-Cadherin. This data suggest that AURKA expression/activity was not directly involved in lung cancer epithelial-mesenchimal transition. The E-Cadherin gene expression (Additional file 1 Figure S1. Panel B) is not increased in the H522 cell line either by AURKA transcript silencing, by siRNA technology, or by AURKA enzymatic inhibition, using the specific inhibitor PHA-739358. Moreover, the inhibition of AURKA activity does not modify the cellular migration of H522 and Calu1 NSCLC cell lines, while stimulates the $\mathrm{H} 1299$ cell line motility (*p $<0.05$, Additional file 1 Figure S1. Panel C). This data suggest that in invasive lung cancer cell lines the cellular motility is not directly dependent on AURKA activity and likely its role in invasion could be affected by molecular cancer micro-environment. Further studies are required to investigate if this behavior is shared also by different NSCLC subtypes in vivo and if it may be utilized to select the optimal therapeutic approach of lung cancer subtypes.

\section{Conclusion}

In this study we reported for the first time that NSCLC histological subtypes showed a different degree of AURKA modulation with the highest over-expression observed in SQC and LCC whereas no significant modulation in BAC was reported. We also identified that the AURKA transcript over-expression was significantly associated to tumor de-differentiation, and its activity was not directly associated to either epithelial marker expression or to enhanced cell motility.

Overall, this data supports the emerging network among genomic instability, AURKA over-expression and tumor progression in NSCLC. Further studies are required to elucidate its involvement in chemotherapeutic resistance as its reliability as a putative predictive marker of personalized NSCLC treatments responsiveness.

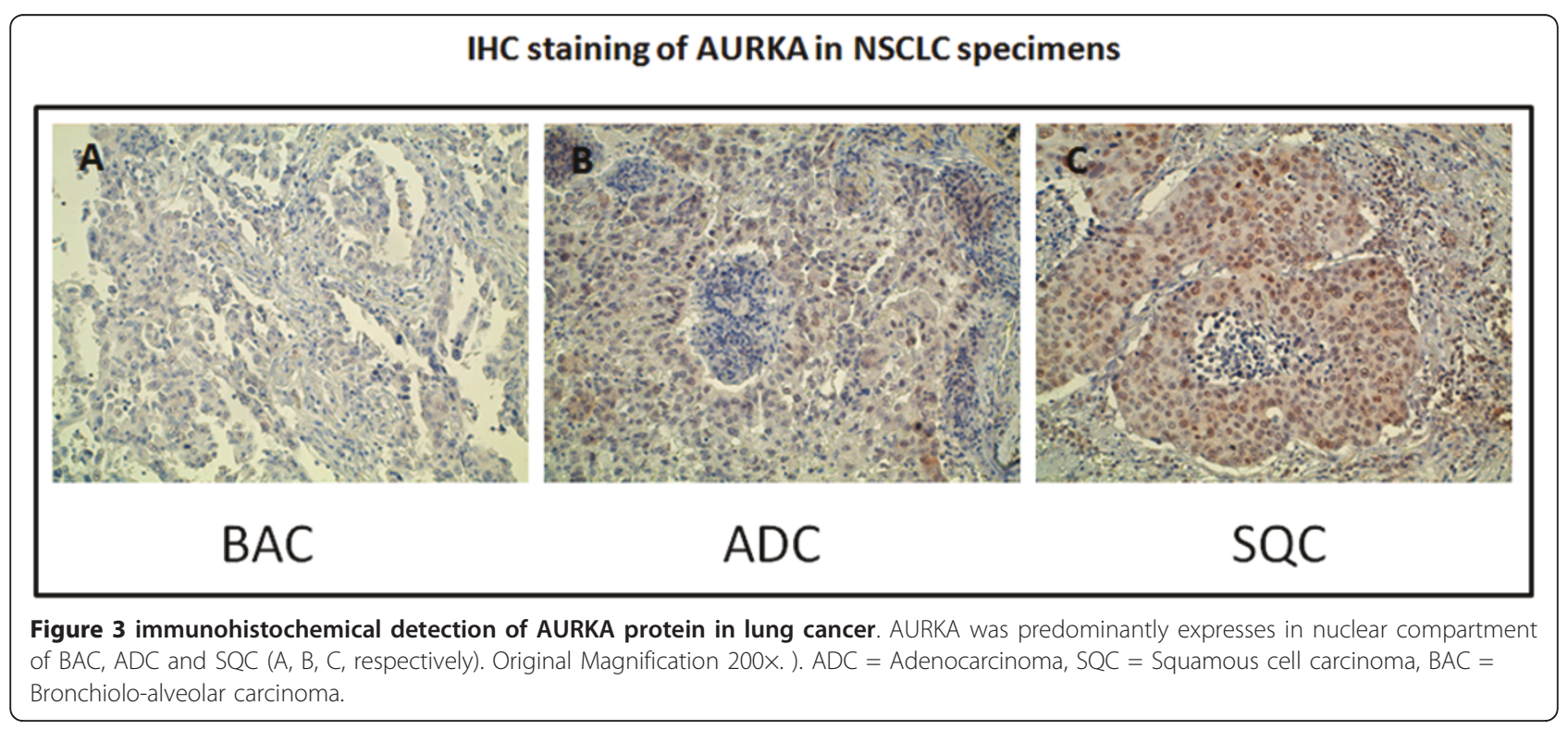




\section{Additional material}

Additional file 1: AURKA expression/activity does not influence migration or Epithelial marker expression in lung cancer cell lines. Figure S1. AURKA expression/activity does not influence migration or Epithelial marker expression in lung cancer cell lines. A) The inhibition of AURKA activity, by the specific inhibitor PHA-739358, weakly increases expression of E-cadherin evaluated by FACS analysis. The highest and the lowest differences between treated and untreated cells were identified in H522 and Calu1, respectively. B) In H522 cell line the inhibition of AURKA expression by specific siRNA for different time conditions does not affect the E-Cadherin gene regulation (left graph). Moreover, the same results were obtained evaluating $E$-Cadherin transcript expression after treating of the $\mathrm{H} 522$ cell line for $24 \mathrm{~h}$ with different concentration of Aurora Kinase inhibitor (PHA-739358) (right graph). C) The inhibition of AURKA activity, by the specific inhibitor PHA-739358, does not modify the normal cellular migration of $\mathrm{H} 522$ and Calu1, while stimulate significantly the H1299 cell line mobility $\left({ }^{*} p<0.05\right)$.

Additional file 2: Additional Materials and Methods. Figure S1 experimental procedure.

\section{Acknowledgements}

The study was supported, in part, by the University of Turin. ML belongs to the fellowship of Regione Piemonte. Special thanks Giuseppe Schiavello for the critical review of manuscript. The authors would like to thank all the members of our clinical collaborators at the San Luigi Hospital involved in this study for their support in facilitating lung cancer specimens and their clinical follow-up.

\section{Authors' contributions}

ML participated in acquiring clinical and laboratory data, data analysis and interpretation, acquiring clinical samples, follow-up clinical information and final writing of the manuscript. VM, SS, PC and EB participated in acquiring clinical and laboratory data, data analysis and data interpretation and drafted the manuscript. MP and GVS participated in study design and coordination, data analysis and interpretation and drafted the manuscript. All authors read and approved the final manuscript.

\section{Competing interests}

The authors declare that they have no competing interests.

Received: 7 October 2010 Accepted: 30 June 2011

Published: 30 June 2011

\section{References}

1. Bischoff JR, Plowman GD: The Aurora/Ipl1p kinase family: regulators of chromosome segregation and cytokinesis. Trends Cell Biol 1999, 9:454-459.

2. Zhou H, Kuang J, Zhong L, Kuo WL, Gray JW, Sahin A, Brinkley BR, Sen S: Tumour amplified kinase STK15/BTAK induces centrosome amplification, aneuploidy and transformation. Nat Genet 1998, 20:189-193.

3. Gritsko TM, Coppola D, Paciga JE, Yang L, Sun M, Shelley SA, Fiorica JV, Nicosia SV, Cheng JQ: Activation and overexpression of centrosome kinase BTAK/Aurora-A in human ovarian cancer. Clin Cancer Res 2003, 9:1420-1426

4. Pihan GA, Purohit A, Wallace J, Malhotra R, Liotta L, Doxsey SJ: Centrosome defects can account for cellular and genetic changes that characterize prostate cancer progression. Cancer Res 2001, 61:2212-2219.

5. Sakakura C, Hagiwara A, Yasuoka R, Fujita Y, Nakanishi M, Masuda K, Shimomura K, Nakamura Y, Inazawa J, Abe T, Yamagishi H: Tumouramplified kinase BTAK is amplified and overexpressed in gastric cancers with possible involvement in aneuploid formation. Br J Cancer 2001, 84:824-831.

6. Sen S, Zhou H, Zhang RD, Yoon DS, Vakar-Lopez F, Ito S, Jiang F, Johnston D, Grossman HB, Ruifrok AC, Katz RL, Brinkley W, Czerniak B: Amplification/overexpression of a mitotic kinase gene in human bladder cancer. J Natl Cancer Inst 2002, 94:1320-1329.
7. Tanaka T, Kimura M, Matsunaga K, Fukada D, Mori H, Okano Y: Centrosomal kinase AIK1 is overexpressed in invasive ductal carcinoma of the breast. Cancer Res 1999, 59:2041-2044.

8. Xu HT, Ma L, Qi FJ, Liu Y, Yu JH, Dai SD, Zhu JJ, Wang EH: Expression of serine threonine kinase 15 is associated with poor differentiation in lung squamous cell carcinoma and adenocarcinoma. Pathol Int 2006, 56:375-380.

9. Liu Q, Kaneko S, Yang L, Feldman RI, Nicosia SV, Chen J, Cheng JQ: AuroraA abrogation of p53 DNA binding and transactivation activity by phosphorylation of serine 215. J Biol Chem 2004, 279:52175-52182.

10. Wang R, Wang JH, Chu XY, Geng HC, Chen LB: Expression of STK15 mRNA in hepatocellular carcinoma and its prognostic significance. Clin Biochem 2009, 42:641-647.

11. Reiter R, Gais P, Jutting U, Steuer-Vogt MK, Pickhard A, Bink K, Rauser S, Lassmann S, Hofler H, Werner M, Walch A: Aurora kinase A messenger RNA overexpression is correlated with tumor progression and shortened survival in head and neck squamous cell carcinoma. Clin Cancer Res 2006, 12:5136-5141.

12. Wan $X B$, Long ZJ, Yan $M$, X J J, Xia LP, Liu L, Zhao Y, Huang XF, Wang XR, Zhu XF, Hong MH, Liu Q: Inhibition of Aurora-A suppresses epithelialmesenchymal transition and invasion by downregulating MAPK in nasopharyngeal carcinoma cells. Carcinogenesis 2008, 29:1930-1937.

13. Travis WD, Brambilla E, Müller-Hermelink HK, Harris CC: World Health Organization classification of tumors: pathology and genetics of tumors of the lung, pleura, thymus and heart Lyon: IARC Press; 2004.

14. Sobin LH, Wittekind C: TNM Classification of Malignant Tumours New York: Wiley-Liss; 2002.

15. R: A language and environment for statistical computing. Vienna, Austria: R Foundation for Statistical Computing;, 8.02009.

16. Tammemagi MC, McLaughlin JR, Bull SB: Meta-analyses of p53 tumor suppressor gene alterations and clinicopathological features in resected lung cancers. Cancer Epidemiol Biomarkers Prev 1999, 8:625-634.

17. Meraldi P, Honda R, Nigg EA: Aurora-A overexpression reveals tetraploidization as a major route to centrosome amplification in p53-/cells. EMBO J 2002, 21:483-492.

18. Tonon G, Brennan C, Protopopov A, Maulik G, Feng B, Zhang Y, Khatry DB, You MJ, Aguirre AJ, Martin ES, Yang Z, Ji H, Chin L, Wong KK, Depinho RA: Common and contrasting genomic profiles among the major human lung cancer subtypes. Cold Spring Harb Symp Quant Biol 2005, 70:11-24.

19. Guarino M, Rubino B, Ballabio G: The role of epithelial-mesenchymal transition in cancer pathology. Pathology 2007, 39:305-318.

20. Bremnes RM, Veve R, Hirsch FR, Franklin WA: The E-cadherin cell-cell adhesion complex and lung cancer invasion, metastasis, and prognosis. Lung Cancer 2002, 36:115-124.

doi:10.1186/1479-5876-9-100

Cite this article as: Lo lacono et al: Aurora Kinase A expression is associated with lung cancer histological-subtypes and with tumor dedifferentiation. Journal of Translational Medicine 2011 9:100.

\section{Submit your next manuscript to BioMed Central and take full advantage of:}

- Convenient online submission

- Thorough peer review

- No space constraints or color figure charges

- Immediate publication on acceptance

- Inclusion in PubMed, CAS, Scopus and Google Scholar

- Research which is freely available for redistribution

Submit your manuscript at www.biomedcentral.com/submit
C Biomed Central 